\title{
Effect of Heterogeneity on Imbibition Phenomena in Fluid Flow through Porous Media with Different Porous Materials
}

https://doi.org/10.1515/nleng-2017-0122

Received September 22, 2017; revised March 4, 2018; accepted March 25, 2018.

\begin{abstract}
In this paper, the counter - current imbibition phenomena in a heterogeneous porous media is studied with the consideration of two types of porous materials like volcanic and fine sand and Adomian decomposition method is applied to find the saturation of wetting phase and the recovery rate of the reservoir. A simulation result is developed here to study the effect of heterogeneity, capillarity and relative permeability on saturation rate and to obtain an optimum recovery rate of the reservoir with the choices of some interesting parametric value.
\end{abstract}

Keywords: Counter - Current Imbibition, Heterogeneous Porous Media, Brooks - Corey model, Adomian decomposition method

MSC: 76S05, 74R10

\section{Introduction}

This paper discusses imbibition phenomena in a heterogeneous porous media with the consideration of two different porous materials like volcanic and fine sand.When the reservoir oil (non - wetting phase) comes into contact with water (wetting phase) then there is a spontaneous flow of the wetting phase (Water) into the medium and a counter flow of the resident fluid i.e. non wetting phase (oil) from the medium initiated by imbibition due to the differences in viscosities of water and oil.Many researchers studied this phenomenon with different approaches.

Hardik S. Patel, Department of Mathematics, Uka Tarsadia University, Maliba Campus, Bardoli-Mahuva Road, Tarsadi, Barodli, Gujarat 394350, India, E-mail: hardy.nit@gmail.com

*Corresponding Author: Ramakanta Meher, Department of Applied Mathematics and Humanities, S. V. National Institute of Technology, Surat, Gujarat, India, E-mail: meher_ramakanta@yahoo.com
Aronofsky et al. [2], first proposed an empirical function

$$
R=R_{\infty}\left(1-e^{-y T}\right)
$$

to study the recovery rate of the reservoir, where $T=$ $\frac{K p_{d}}{\phi \mu_{w} L^{2}} t$ be the dimensionless time used for studying the recovery rate with dimensionless time.

Meng, Liu \& Wang [8] presented a critical review on the fundamental mechanisms of spontaneous imbibition, counter - current imbibition and the impact of boundary condition, fluid viscosity and wettability on co-current and counter current imbibition. Kashchiev and Firoozabadi [6] presented an analytical study for one dimensional counter - current flow of water and oil in porous media and obtained an expression for the time dependence of the water saturation profile and for the oil recovered during spontaneous counter - current imbibition. Pooladi - Darvish and Firoozabadi [11] predicted experimentally the performance of water injection in a naturally fractured reservoirs with the consideration of a number of water wet matrix blocks and revealed that the dominant recovery mechanism changed from co - current to counter - current imbibition when the boundary conditions changed from advancing FWL to immersion type and concluded by performing a single block experiments of co - current and counter - current imbibition that the co - current imbibition leads to faster oil recovery as compared to counter current imbibition. Saboorian - Jooybari, Ashoori, and Mowazi [17] studied the fluid saturation distribution analytically in a fractured reservoir within a matrix block and derived the saturation profiles by solving the capillary - diffusion equation under different imposed boundary conditions for the processes where counter - current imbibition is the dominant oil drive mechanism. Saboorian - Jooybari, H., Ashoori, S., and Mowazi, G.H. [18] formulated the three - dimensional distribution of fluid saturation within a matrix block and time-dependent matrix fracture shape factor formulation and analytically derived the saturation profiles by solving the saturation diffusion equation in the counter - current imbibition process by including both capillary and gravity forces.

Zeybek et al. [19] performed a numerical simulation and studied the capillary imbibition in porous structures with 
the effect of heterogeneity, permeability and wettability on counter - current and co - current imbibition. Hughes and Blunt [4] found that the pattern of displacement and the rate of imbibition depends on the relationship between capillary number, contact angle and initial wetting phase saturation. Pooladi - Darvish and Firoozabadi [10] modelled the co - and counter - current imbibition in water - wet rocks and found that the flow is dominated by co - current imbibition when the porous media are partially covered by water, and concluded that the oil recovery from co - current imbibition is higher than the one from the counter - current. similarly, Karpynet al. [5] identified three distinct flow intervals in spontaneous imbibition in a layered sandstone with a single longitudinal fracture, where counter - current flow was dominant at early and intermediate times; while both co - current and counter - current flow mechanisms coexisted at late times. Meher et al. [7] discussed this phenomenon in a homogeneous porous media with the consideration of capillary pressure and concluded that the saturation of water increases exponentially with distance $X$ for any time $T>0$ while Patel et al. [16] studied this phenomenon in a heterogeneous porous media and concluded that the saturation rate be more in homogeneous porous matrix as compared to heterogeneous porous matrix. Similarly Patel and Meher [12] studied the Fingering phenomena in a fractured porous media with the consideration of inclination and gravitational Effect and developed a simulation result for the saturation of wetting phase with and without considering the inclination effect and concluded that the saturation of wetting phase be increases with time and it is more for zero and small inclination while (Patel and Meher [15], Meher $[13,14])$ considered Corey's model and discussed this phenomenon in a heterogeneous porous medium with capillary pressure and in an inclined heterogeneous porous media with the consideration different porous materials.

Here the main objective of this work is to study the effects of two different types of porous materials like volcanic and fine sand on initial water saturation and the effects of capillary pressure, porosity and relative permeability on saturation rate during imbibition phenomena in fluid flow through heterogeneous porous media. Analytical solution for the flow equations has been obtained by using Adomian decomposition method to study the saturation of wetting phase and a simulation result is developed here to study the recovery rate of the reservoir.The effect of capillary pressure, porosity and relative permeability on saturation rate can be verified from the expression obtained for saturation.It is of great significance in oil recovery, where it can be responsible to increase the oil production up to $40 \%-50 \%$ in some cases.

\section{Mathematical model}

For the sake of mathematical model: here it is considered a piece of porous matrix of an oil formatted region of length ' $L$ ' having heterogeneity in pore size distribution that contains viscous oil and is completely surrounded by an impermeable surface except for one end (common interface) that is labelled as the Imbibition face and it is exposed to an adjacent formation of 'injected' water. Due to the differences in viscosities of water and oil, the water saturates on the right side of imbibition face and it travel only a small distance ' $l$ ' due to the capillary pressure effect(without external force) initiated by imbibition as shown in fig-1.

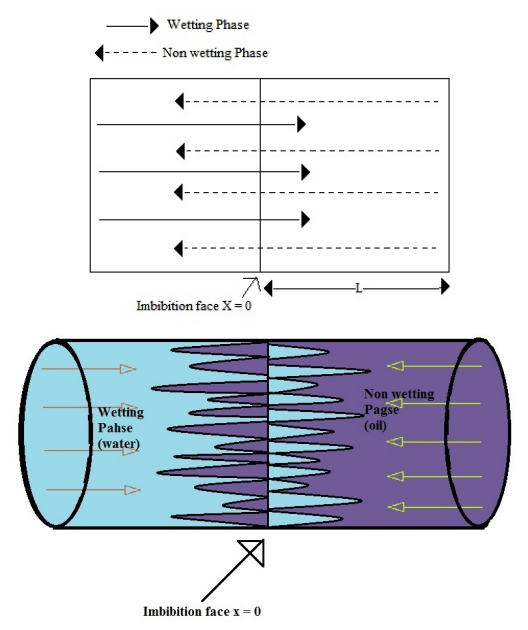

Fig. 1: Schematic diagram of the problem under consideration

The conservation equation of mass for two phase flow with respect to volume can be formulated as

$$
\frac{\partial}{\partial t}\left(\phi(x) S_{i} \rho_{i}\right)+\nabla \cdot\left(v_{i} \rho_{i}\right)=0
$$

Where $i=o, w, x \in \Re^{3}, t \geq 0, \phi(x)$ denotes the porosity of the porous medium, $S_{i}$ is the saturation for each phase $i$, $\rho_{i}$ is its specific mass and $v_{i}$ is the Darcy velocity, given by

$$
v_{i}=-K(x) \frac{k_{i}}{\mu_{i}}\left(\nabla p_{i}\right)
$$

Where $K(x)$ denotes the absolute permeability tensor of the porous medium, $p_{i}$ is its pressure, $k_{i}$ is its relative permeability, and $\mu_{i}$ is its viscosity.

If the compressibility of the injected fluid is neglected, then $\rho$ 's are constant and the conservation equation becomes

$$
\frac{\partial}{\partial t}\left(\phi(x) S_{i}\right)+\nabla \cdot v_{i}=0, \quad i=o, w
$$


The condition for seepage velocity and capillary pressure in counter - current imbibition can be expressed (Patel et al. [16]) as

$$
\begin{gathered}
v_{w}=-v_{o} \\
P_{c}=p_{o}-p_{w}
\end{gathered}
$$

According to Oroveanu [9] and Patel et al. [16], the laws of variation in the porosity and permeability of a uniform heterogeneous medium can be defined as a function of $x$ only and for the definiteness of the problem, it can be defined as

$$
\begin{aligned}
\phi(x) & =\frac{1}{a-b x} \\
K(x) & =K_{c} \phi(x)
\end{aligned}
$$

Where $a, b$ and $K_{c}$ are constants.

The most famous $P_{c}-S_{w}$ relationships (Brooks and Corey [3]) can be expressed as

$$
p_{c}\left(S_{w}\right)=p_{d} S_{e}^{-\frac{1}{\lambda}}=p_{d}\left(\frac{S_{w}-S_{w r}}{1-S_{w r}}\right)^{-\frac{1}{\lambda}}
$$

Similarly, the relative permeability, $k_{w}$ of the wetting and non - wetting phases in the domain are governed by the following equation

$$
k_{0}=S_{e}^{\frac{2+3 \lambda}{\lambda}}
$$

Combining eq. (3), (5) and (6), it obtains

$$
v_{w}=K(x) \frac{k_{o} k_{w}}{k_{w} \mu_{o}+k_{o} \mu_{w}}\left[\frac{\partial p_{c}}{\partial x}\right]
$$

Hence the conservation eq. (4) with eq. (10) can be written as

$$
\phi \frac{\partial S_{w}}{\partial t}+\frac{\partial}{\partial x}\left[K(x) \frac{k_{o} k_{w}}{k_{w} \mu_{o}+k_{o} \mu_{w}} \frac{\partial p_{c}}{\partial S_{w}} \frac{\partial S_{w}}{\partial x}\right]=0
$$

By using eq. (8) and (9) with eq. (11), it yields

$$
\begin{aligned}
& \phi \frac{\partial S_{w}}{\partial t}+\frac{K_{c} p_{d}}{\mu_{0}} \frac{\partial}{\partial x} \\
& {\left[\phi\left(\frac{S_{w}-S_{w r}}{1-S_{w r}}\right)^{\frac{2+3 \lambda}{\lambda}} \frac{\partial}{\partial S_{w}}\left(\frac{S_{w}-S_{w r}}{1-S_{w r}}\right)^{-\frac{1}{\lambda}} \frac{\partial S_{w}}{\partial x}\right]=0}
\end{aligned}
$$

Where $\frac{k_{o} k_{w}}{k_{w} \mu_{o}+k_{o} \mu_{w}} \approx \frac{k_{0}}{\mu_{0}}$ (Patel et al. [16]) Simplifying eq. (12), it becomes

$$
\begin{aligned}
& \frac{\partial S_{w}}{\partial t}+\frac{K_{c} p_{d}}{\mu_{0}} \\
& {\left[\frac{\partial}{\partial x}\left(\left(\frac{S_{w}-S_{w r}}{1-S_{w r}}\right)^{\frac{2+3 \lambda}{\lambda}} \frac{\partial}{\partial S_{w}}\left(\frac{S_{w}-S_{w r}}{1-S_{w r}}\right)^{-\frac{1}{\lambda}} \frac{\partial S_{w}}{\partial x}\right)\right.}
\end{aligned}
$$

$$
\left.+\left(\frac{S_{w}-S_{w r}}{1-S_{w r}}\right)^{\frac{2+3 \lambda}{\lambda}} \frac{\partial}{\partial S_{w}}\left(\frac{S_{w}-S_{w r}}{1-S_{w r}}\right)^{-\frac{1}{\lambda}} \frac{\partial S_{w}}{\partial x} \frac{1}{\phi} \frac{\partial \phi}{\partial x}\right]=0
$$

Using the dimensionless variables

$$
X=\frac{x}{L} \quad \text { and } \quad T=\frac{K_{c} p_{d}}{\mu_{o} L^{2}} t
$$

And simplification of $\frac{1}{\phi} \frac{\partial \phi}{\partial X}$ as

$$
\frac{1}{\phi} \frac{\partial \phi}{\partial X}=\frac{\partial}{\partial X}(\log \phi)=\frac{\partial}{\partial X}\left[\frac{b}{a} L X-\log a\right]
$$

(Neglecting higher order term of X)

$$
=\frac{b L}{a}
$$

It gives the dimensionless forms of eq. (13) as

$$
\begin{aligned}
& \frac{\partial S_{w}}{\partial T}+\frac{\partial}{\partial X}\left[\left(\frac{S_{w}-S_{w r}}{1-S_{w r}}\right)^{\frac{2+3 \lambda}{\lambda}} \frac{\partial}{\partial S_{w}}\left(\frac{S_{w}-S_{w r}}{1-S_{w r}}\right)^{-\frac{1}{\lambda}} \frac{\partial S_{w}}{\partial X}\right] \\
& +\frac{b L}{a}\left(\frac{S_{w}-S_{w r}}{1-S_{w r}}\right)^{\frac{2+3 \lambda}{\lambda}} \frac{\partial}{\partial S_{w}}\left(\frac{S_{w}-S_{w r}}{1-S_{w r}}\right)^{-\frac{1}{\lambda}} \frac{\partial S_{w}}{\partial X}=0
\end{aligned}
$$

Eq. (15) describes the equation of imbibition phenomena in a heterogeneous porous media for two different porous materials for different values of $\lambda$.

\section{Analysis of the Method}

For the purpose of illustration of the Adomian decomposition method (Adomian [1]), we consider eq. (15) in an operator form as

$$
L_{T} S_{w}(X, T)+L_{X}\left(N S_{w}\right)+\frac{b L}{a}\left[N S_{w}\right]=0
$$

Where $N S_{w}=\left(\frac{S_{w}-S_{w r}}{1-S_{w r}}\right)^{\frac{2+3 \lambda}{\lambda}} \frac{\partial}{\partial S_{w}}\left(\frac{S_{w}-S_{w r}}{1-S_{w r}}\right)^{-\frac{1}{\lambda}} \frac{\partial S_{w}}{\partial X}$ and $S_{w 0}$ can be found from the initial condition $S(X, 0)=f(X)=e^{-X}$. Operating the inverse operator $L_{T}^{-1}$ and by using the analysis of Adomian decomposition method, the recursive relation of eq. (16) can be expressed as

$$
\begin{aligned}
& \sum_{n=0}^{\infty} S_{w n}(X, T)=e^{-X}+L_{T}^{-1}\left[L_{X}\left(\sum_{n=0}^{\infty} A_{n}\right)\right] \\
& +\frac{b L}{a} L_{T}^{-1}\left[\sum_{n=0}^{\infty} A_{n}\right]
\end{aligned}
$$

This gives the recurrence relation as

$$
\begin{aligned}
S_{w 0} & =S_{w}(X, 0)=e^{-X} \\
S_{w, k+1} & =L_{T}^{-1}\left[L_{X}\left(A_{k}\right)\right]+\frac{b L}{a}\left(A_{k}\right), k \geq 0
\end{aligned}
$$


Using eq. (18), an approximate analytical solution of eq.(15) can be written in series form up to four terms as

$S_{w}(X, T)=S_{w 0}+S_{w 1}+S_{w 2}+\cdots$

$S_{w}(X, T)=e^{-X}+\frac{(b L \lambda-3 a \lambda-\lambda)\left(e^{-X}\right)^{\frac{1}{\lambda}} e^{-3 X}}{\lambda^{2}\left(1-S_{w r}\right) a} T$

$+\frac{\left(e^{-X}\right) \frac{2}{\lambda} e^{-5 X}\left(\begin{array}{l}5 b^{2} L^{2} \lambda^{3}+2 b^{2} L^{2} \lambda^{2}-40 b L a \lambda^{3}-31 b L a \lambda^{2} \\ +75 a^{2} \lambda^{3}-6 b L a \lambda+85 a^{2} \lambda^{2}+32 a^{2} \lambda+4 a^{2}\end{array}\right)}{\lambda^{5}\left(1-S_{w r}\right)^{2} a^{2}} \frac{T^{2}}{2 !}$

Eq. (19) represents the saturation of wetting phase during counter - current imbibition phenomena in a heterogeneous porous media for two different porous materials for different values of where the heterogeneity of the porous medium depends upon the values $\frac{b L}{a}$ since the porosity $\phi$ has been approximated by $\frac{b L}{a}$ in eq. (14).

Table 1: Parametric value of parameters (Brooks and Corey [3])

\begin{tabular}{lccccc}
\hline Property & $\lambda$ & $S_{w r}$ & $p_{d}\left(N / m^{2}\right)$ & $K\left(\mu^{2}\right)$ & $\phi$ \\
\hline Volcanic sand & 2.29 & 0.157 & 16 & 18 & 0.351 \\
Fine sand & 3.70 & 0.167 & 41 & 2.5 & 0.377 \\
\hline
\end{tabular}

\section{Numerical results and Discussion}

\subsection{Effect of inclination on initial water saturation}

Fig. 2 and 3 discusses the variation of saturation rate in heterogeneous porous media in volcanic and fine sand at a fixed distance $X=0.5$. It shows that the saturation rate be more in homogeneous porous media as well as in volcanic sand as compared to heterogeneous porous media and in fine sand.The numerical values of the saturation rate have been discussed in Table 2 to Table 5 .

\subsection{Effect of capillary pressure and heterogeneity on saturation rate}

Fig. 5 discusses the variation of capillary pressure and heterogeneity on saturation rate in volcanic and fine sand. It shows that the effect of capillary pressure be more in heterogeneous as compared to homogeneous porous media

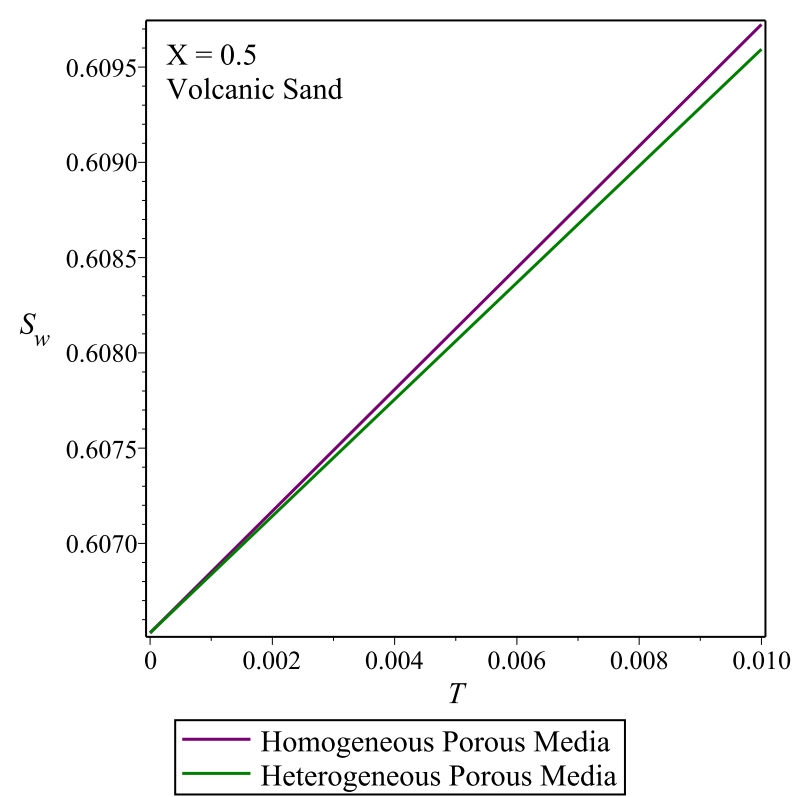

Fig. 2: Saturation of water vs. Dimensionless time

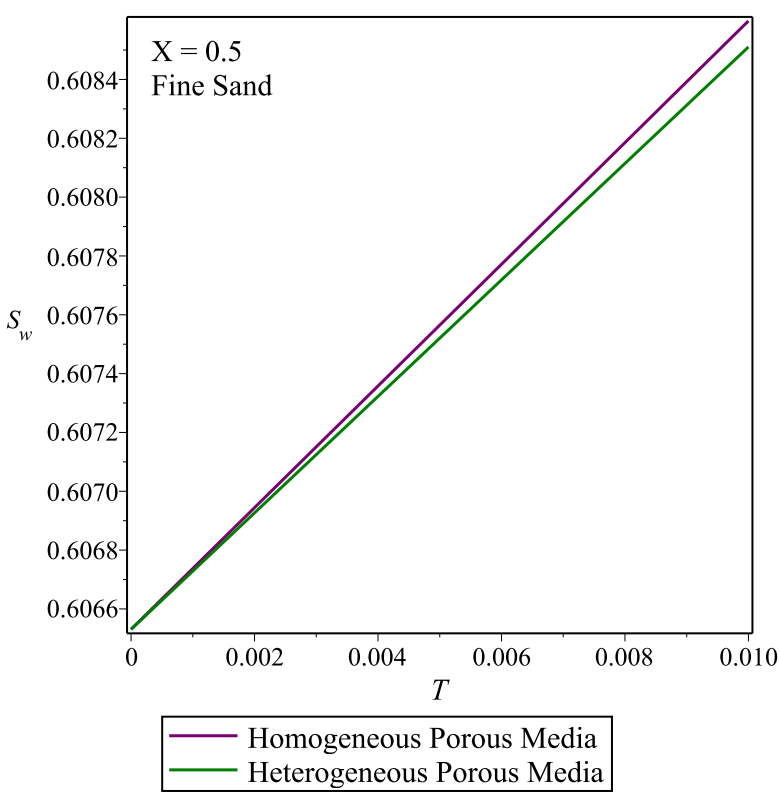

Fig. 3: Saturation of water vs. Dimensionless time

and in fine sand.The numerical value of the saturation rate has been discussed in Table 6 .

\subsection{Effect of relative permeability on saturation rate}

Fig. 7 discusses the variation of relative permeability on saturation rate in volcanic and in fine sand. It shows that 

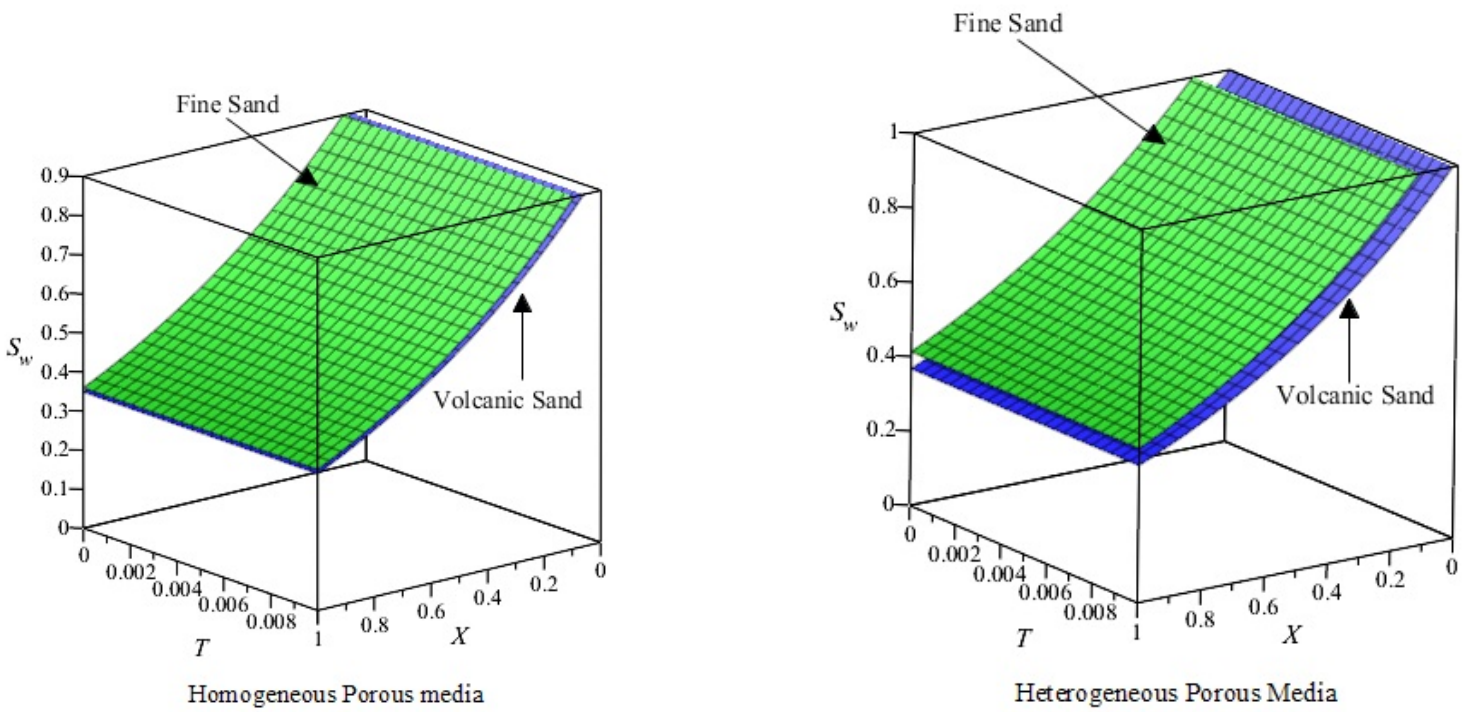

Fig. 4: Saturation of water in Volcanic Sand and Fine Sand for Homogeneous and Heterogeneous Porous Media

Table 2: Numerical values of Saturation ratein Volcanic Sand in Homogeneous Porous Media

\begin{tabular}{ccccccccccc}
\hline & \multicolumn{1}{c}{ Volcanic Sand } \\
$S_{w}(X, T)$ & $T=0.001$ & $T=0.002$ & $T=0.003$ & $T=0.004$ & $T=0.005$ & $T=0.006$ & $T=0.007$ & $T=0.008$ & $T=0.009$ & $T=0.010$ \\
\hline 0.1 & 0.906099 & 0.907362 & 0.908624 & 0.909887 & 0.911149 & 0.912412 & 0.913674 & 0.914937 & 0.916199 & 0.917462 \\
0.2 & 0.819626 & 0.820521 & 0.821416 & 0.822311 & 0.823207 & 0.824102 & 0.824997 & 0.825893 & 0.826788 & 0.827683 \\
0.3 & 0.741453 & 0.742088 & 0.742722 & 0.743357 & 0.743992 & 0.744627 & 0.745262 & 0.745897 & 0.746532 & 0.747167 \\
0.4 & 0.670770 & 0.671220 & 0.671670 & 0.672121 & 0.672571 & 0.673021 & 0.673471 & 0.673922 & 0.674372 & 0.674822 \\
0.5 & 0.606849 & 0.607169 & 0.607488 & 0.607807 & 0.608127 & 0.608446 & 0.608765 & 0.609085 & 0.609404 & 0.609723 \\
0.6 & 0.549038 & 0.549264 & 0.549490 & 0.549717 & 0.549943 & 0.550170 & 0.550396 & 0.550623 & 0.550849 & 0.551076 \\
0.7 & 0.496745 & 0.496906 & 0.497067 & 0.497227 & 0.497388 & 0.497548 & 0.497709 & 0.497869 & 0.498030 & 0.498191 \\
0.8 & 0.449442 & 0.449556 & 0.449670 & 0.449784 & 0.449898 & 0.450012 & 0.450126 & 0.450240 & 0.450353 & 0.450467 \\
0.9 & 0.406650 & 0.406731 & 0.406811 & 0.406892 & 0.406973 & 0.407054 & 0.407134 & 0.407215 & 0.407296 & 0.407377 \\
1.0 & 0.367936 & 0.367993 & 0.368051 & 0.368108 & 0.368165 & 0.368223 & 0.368280 & 0.368337 & 0.368394 & 0.368452 \\
\hline
\end{tabular}

Table 3: Numerical values of Saturation rate in Volcanic Sand in Heterogeneous Porous Media

\begin{tabular}{ccccccccccc}
\hline & \multicolumn{1}{c}{ Volcanic Sand } \\
$S_{w}(X, T)$ & $T=0.001$ & $T=0.002$ & $T=0.003$ & $T=0.004$ & $T=0.005$ & $T=0.006$ & $T=0.007$ & $T=0.008$ & $T=0.009$ & $T=0.010$ \\
\hline 0.1 & 0.906048 & 0.907259 & 0.908470 & 0.909681 & 0.910892 & 0.912103 & 0.913314 & 0.914525 & 0.915736 & 0.916947 \\
0.2 & 0.819589 & 0.820448 & 0.821307 & 0.822166 & 0.823024 & 0.823883 & 0.824742 & 0.825601 & 0.826460 & 0.827319 \\
0.3 & 0.741427 & 0.742036 & 0.742645 & 0.743254 & 0.743863 & 0.744472 & 0.745081 & 0.745690 & 0.746299 & 0.746908 \\
0.4 & 0.670751 & 0.671183 & 0.671615 & 0.672047 & 0.672479 & 0.672911 & 0.673343 & 0.673775 & 0.674207 & 0.674639 \\
0.5 & 0.606836 & 0.607143 & 0.607449 & 0.607755 & 0.608062 & 0.608368 & 0.608674 & 0.608981 & 0.609287 & 0.609593 \\
0.6 & 0.549028 & 0.549246 & 0.549463 & 0.549680 & 0.549897 & 0.550114 & 0.550332 & 0.550549 & 0.550766 & 0.550983 \\
0.7 & 0.496739 & 0.496893 & 0.497047 & 0.497201 & 0.497355 & 0.497509 & 0.497663 & 0.497817 & 0.497971 & 0.498125 \\
0.8 & 0.449438 & 0.449547 & 0.449656 & 0.449765 & 0.449875 & 0.449984 & 0.450093 & 0.450202 & 0.450312 & 0.450421 \\
0.9 & 0.406647 & 0.406724 & 0.406802 & 0.406879 & 0.406957 & 0.407034 & 0.407111 & 0.407189 & 0.407266 & 0.407344 \\
1.0 & 0.367934 & 0.367989 & 0.368044 & 0.368099 & 0.368154 & 0.368209 & 0.368264 & 0.368318 & 0.368373 & 0.407344 \\
\hline
\end{tabular}

the effect of Relative permeability is more in homogeneous porous media as compared to heterogeneous porous me- dia and in volcanic sand.The numerical values of the saturation rate has been discussed in Table 7. 
Table 4: Numerical values of Saturation rate in Fine Sand in Homogeneous Porous Media

\begin{tabular}{|c|c|c|c|c|c|c|c|c|c|c|}
\hline$S_{w}(X, T)$ & $T=0.001$ & $T=0.002$ & $T=0.003$ & $T=0.004$ & $\begin{array}{c}\text { Fine Sand } \\
T=0.005\end{array}$ & $T=0.006$ & $T=0.007$ & $T=0.008$ & $T=0.009$ & $T=0.010$ \\
\hline 0.1 & 0.905602 & 0.906367 & 0.907132 & 0.907897 & 0.908662 & 0.909427 & 0.910193 & 0.910958 & 0.911723 & 0.912488 \\
\hline 0.2 & 0.819282 & 0.819834 & 0.820385 & 0.820937 & 0.821489 & 0.822040 & 0.822592 & 0.823144 & 0.823695 & 0.824247 \\
\hline 0.3 & 0.741216 & 0.741613 & 0.742011 & 0.742409 & 0.742807 & 0.743204 & 0.743602 & 0.744000 & 0.744398 & 0.744796 \\
\hline 0.4 & 0.670606 & 0.670893 & 0.671180 & 0.671467 & 0.671754 & 0.672041 & 0.672327 & 0.672614 & 0.672901 & 0.673188 \\
\hline 0.5 & 0.606737 & 0.606944 & 0.607151 & 0.607357 & 0.607564 & 0.607771 & 0.607978 & 0.608185 & 0.608392 & 0.608598 \\
\hline 0.6 & 0.548960 & 0.549109 & 0.549259 & 0.549408 & 0.549557 & 0.549706 & 0.549855 & 0.550004 & 0.550153 & 0.550302 \\
\hline 0.7 & 0.496692 & 0.496800 & 0.496907 & 0.497015 & 0.497122 & 0.497230 & 0.497338 & 0.497445 & 0.497553 & 0.497660 \\
\hline 0.8 & 0.449406 & 0.449484 & 0.449561 & 0.449639 & 0.449716 & 0.449794 & 0.449871 & 0.449949 & 0.450026 & 0.450104 \\
\hline 0.9 & 0.406625 & 0.406681 & 0.406737 & 0.406793 & 0.406849 & 0.406905 & 0.406961 & 0.407016 & 0.407072 & 0.407128 \\
\hline 1.0 & 0.367919 & 0.367960 & 0.368000 & 0.368040 & 0.368081 & 0.368121 & 0.368161 & 0.368201 & 0.368242 & 0.368282 \\
\hline
\end{tabular}

Table 5: Numerical values of Saturation rate in Fine Sand in Heterogeneous Porous Media

\begin{tabular}{ccccccccccc}
\hline & & \multicolumn{3}{c}{ Fine Sand } \\
$S_{w}(X, T)$ & $T=0.001$ & $T=0.002$ & $T=0.003$ & $T=0.004$ & $T=0.005$ & $T=0.006$ & $T=0.007$ & $T=0.008$ & $T=0.009$ & $T=0.010$ \\
\hline 0.1 & 0.905569 & 0.906302 & 0.907034 & 0.907766 & 0.908499 & 0.909231 & 0.909963 & 0.910696 & 0.911428 & 0.912160 \\
0.2 & 0.819258 & 0.819786 & 0.820314 & 0.820842 & 0.821371 & 0.821899 & 0.822427 & 0.822955 & 0.823483 & 0.824011 \\
0.3 & 0.741198 & 0.741579 & 0.741960 & 0.742341 & 0.742722 & 0.743102 & 0.743483 & 0.743864 & 0.744245 & 0.744625 \\
0.4 & 0.670594 & 0.670869 & 0.671143 & 0.671418 & 0.671692 & 0.671967 & 0.672241 & 0.672516 & 0.672791 & 0.673065 \\
0.5 & 0.606728 & 0.606926 & 0.607124 & 0.607322 & 0.607520 & 0.607718 & 0.607916 & 0.608114 & 0.608312 & 0.608510 \\
0.6 & 0.548954 & 0.549097 & 0.549239 & 0.549382 & 0.549525 & 0.549668 & 0.549810 & 0.549953 & 0.550096 & 0.550239 \\
0.7 & 0.496688 & 0.496791 & 0.496894 & 0.496997 & 0.497099 & 0.497202 & 0.497305 & 0.497408 & 0.497511 & 0.497614 \\
0.8 & 0.449403 & 0.449477 & 0.449551 & 0.449625 & 0.449700 & 0.449774 & 0.449848 & 0.449922 & 0.449996 & 0.450071 \\
0.9 & 0.406623 & 0.406676 & 0.406730 & 0.406783 & 0.406837 & 0.406890 & 0.406944 & 0.406997 & 0.407051 & 0.407104 \\
1.0 & 0.367918 & 0.367956 & 0.367995 & 0.368033 & 0.368072 & 0.368110 & 0.368149 & 0.368188 & 0.368226 & 0.368265 \\
\hline
\end{tabular}

Table 6: Numerical values of capillary pressure vs. saturation in volcanic sand and fine sand

\begin{tabular}{|c|c|c|c|c|c|c|c|}
\hline \multicolumn{8}{|c|}{$T=0.005$} \\
\hline \multicolumn{4}{|c|}{ Volcanic Sand } & \multicolumn{4}{|c|}{ Fine Sand } \\
\hline \multicolumn{2}{|c|}{ Homogeneous Porous Media } & \multicolumn{2}{|c|}{ Heterogeneous Porous Media } & \multicolumn{2}{|c|}{ Homogeneous Porous Media } & \multicolumn{2}{|c|}{ Heterogeneous Porous Media } \\
\hline$\left(S_{w}\right)$ & $\left(p_{c}\right)$ & $\left(S_{w}\right)$ & $\left(p_{c}\right)$ & $\left(S_{w}\right)$ & $\left(p_{c}\right)$ & $\left(S_{w}\right)$ & $\left(p_{c}\right)$ \\
\hline 0.911149796 & 16.797406580 & 0.910892649 & 16.799908290 & 0.908662853 & 42.307355190 & 0.908499087 & 42.309880380 \\
\hline 0.823207266 & 17.769717700 & 0.823024907 & 17.734080460 & 0.821489138 & 43.761545980 & 0.821371052 & 43.763680190 \\
\hline 0.743992804 & 18.739758900 & 0.743863481 & 18.741562080 & 0.742807193 & 45.302955580 & 0.742722046 & 45.304766340 \\
\hline 0.672571347 & 19.832084660 & 0.672479636 & 19.833625360 & 0.671754223 & 46.944554170 & 0.671692826 & 46.946097570 \\
\hline 0.608127202 & 21.022841020 & 0.608062163 & 21.024164670 & 0.607564794 & 48.702373900 & 0.607520522 & 48.703696680 \\
\hline 0.549943846 & 22.329476110 & 0.549897724 & 22.330620740 & 0.549557313 & 50.596594560 & 0.549525391 & 50.597735720 \\
\hline 0.497388227 & 23.774325890 & 0.497355518 & 23.775323570 & 0.497122985 & 52.653129800 & 0.497099967 & 52.654122080 \\
\hline 0.449898368 & 25.386625170 & 0.449875173 & 25.387503140 & 0.449716667 & 54.906019770 & 0.449700069 & 54.906890980 \\
\hline 0.406973461 & 27.205624770 & 0.406957011 & 27.206406590 & 0.406849218 & 57.401177480 & 0.406837250 & 57.401951600 \\
\hline 0.368165803 & 29.285623520 & 0.368154137 & 29.286330020 & 0.368081021 & 60.202516580 & 0.368072391 & 60.203214900 \\
\hline
\end{tabular}

\section{Recovery rate}

Fig. 9 and 110 discusses the variation of saturation rate in volcanic and fine sand which shows that the recovery rate be more in volcanic sand and in homogeneous porous media as compared to heterogeneous porous media and in find sand implies the recovery rate be optimum in the pres- ence of volcanic sand as compared to fine sand.The numerical Recovery rate value for both porous materials has been discussed in Table 8.

It is found here that the dependence of the type of porous materials and the effect of heterogeneity on saturation rate rendered the problem highly nonlinear. The significant part of this study is to study the advantage of the proposed mathematical expression in the determina- 
Table 7: Numerical values of relative permeability vs. saturation in volcanic sand and fine sand

\begin{tabular}{|c|c|c|c|c|c|c|c|}
\hline \multicolumn{8}{|c|}{$T=0.005$} \\
\hline \multicolumn{4}{|c|}{ Volcanic Sand } & \multicolumn{4}{|c|}{ Fine Sand } \\
\hline \multicolumn{2}{|c|}{ Homogeneous Porous Media } & \multicolumn{2}{|c|}{ Heterogeneous Porous Media } & \multicolumn{2}{|c|}{ Homogeneous Porous Media } & \multicolumn{2}{|c|}{ Heterogeneous Porous Media } \\
\hline$\left(S_{w}\right)$ & $\left(k_{w}\right)$ & $\left(S_{w}\right)$ & $\left(k_{w}\right)$ & $\left(S_{w}\right)$ & $\left(k_{w}\right)$ & $\left(S_{w}\right)$ & $\left(k_{w}\right)$ \\
\hline 0.911149796 & 0.649599417 & 0.910892649 & 0.648741896 & 0.908662853 & 0.662858056 & 0.908499087 & 0.662339988 \\
\hline .823207266 & 0.394346473 & 0.823024907 & 0.401431366 & 0.821489138 & 0.425750068 & 0.82137 & 0.425478161 \\
\hline 0.743992804 & 0.246109044 & 0.743863481 & 0.245899092 & 0.742807193 & 0.270527173 & 0.7427 & 0.270385563 \\
\hline 0.672571347 & 0.148897301 & 0.672479636 & 0.148794737 & 0.67175 & 0.169707937 & 0.671 & 862 \\
\hline 0.608127202 & 0.088771074 & 0.608062163 & 0.088721513 & 0.607564794 & 0.104848402 & 0.6075 & $0.104 \varepsilon$ \\
\hline 0.549943846 & 0.051998600 & 0.549897724 & 0.051974963 & 0.549557313 & 0.063603302 & & 0.0635 \\
\hline 0.497388227 & 0.029817032 & 0.497355518 & 0.029805936 & 0.497122985 & 0.037740845 & 0.4970 & 0.037731529 \\
\hline 0.449898368 & 0.016660888 & 0.449875173 & 0.016655778 & 0.449716667 & 0.021799524 & 0.449700069 & 0.021794994 \\
\hline 0.406973461 & 0.009018235 & 0.406957011 & 0.009015936 & 0.406849218 & 0.012178843 & 0.406837250 & 0.012176691 \\
\hline 0.368165803 & 0.004691579 & 0.368154137 & 0.004690575 & 0.368081021 & 0.006524041 & 0.368072391 & 0.006523049 \\
\hline
\end{tabular}
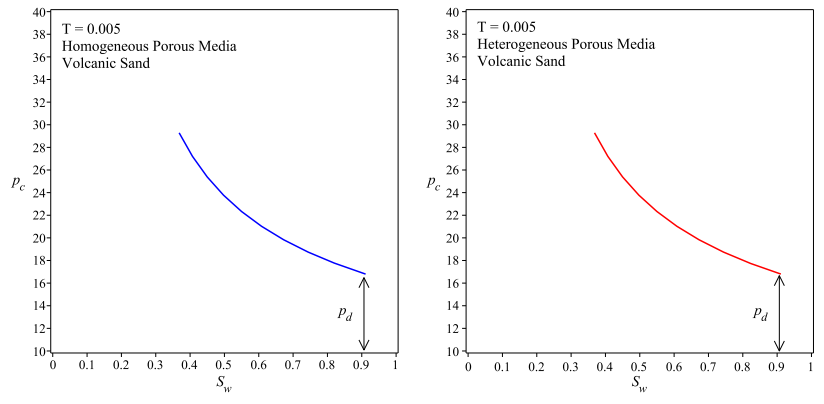

(a)

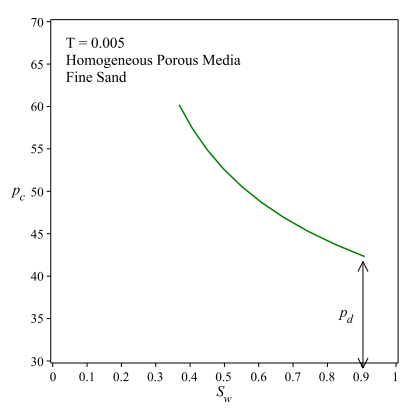

(b)

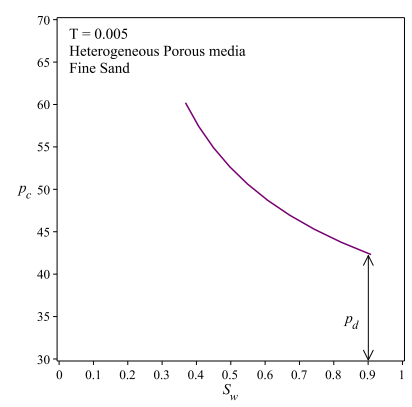

(c)

(d)

Fig. 5: Comparison between capillary pressure vs. Saturation in Volcanic Sand (Fig. (a) and (b)) and Fine Sand (Fig. (c) and (d))

tion of saturation rate and the recovery rate of the reservoir with the effect of heterogeneity and with the consideration of different porous materials with suitable choices of parametric values. It is found that there is an impact of heterogeneity and the types of porous materials on saturation rate and it shows that the saturation rate be more in homogeneous porous media and in volcanic sand as compared to fine sand in heterogeneous porous media.

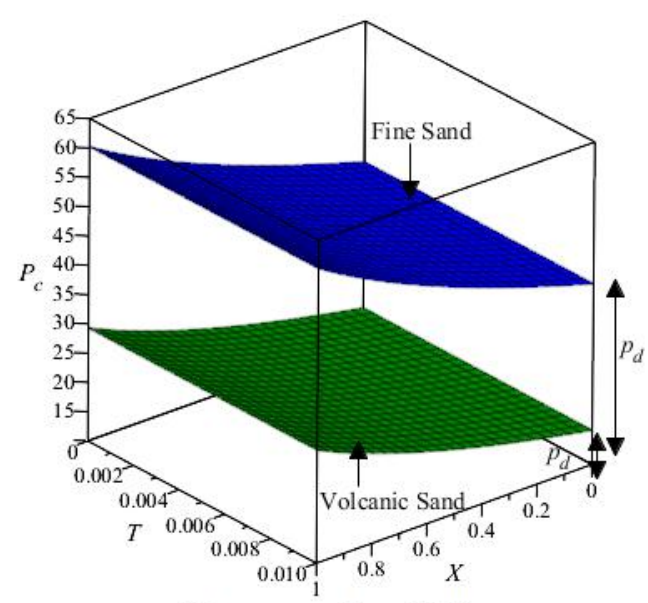

Homogeneous Porous Media

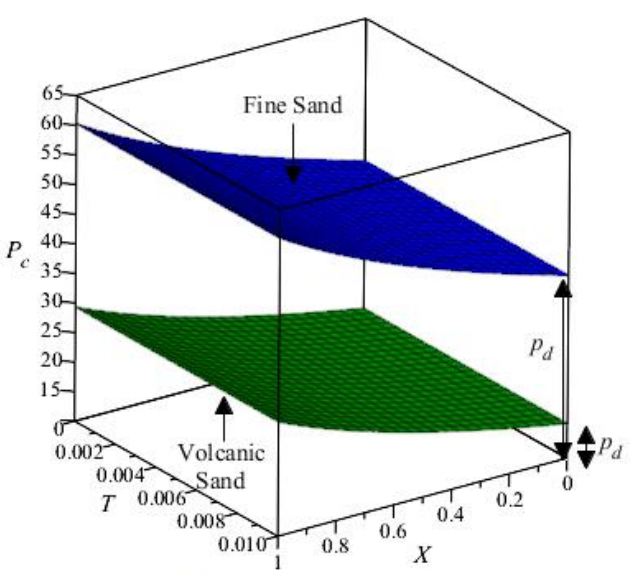

Heterogeneous Porous Media

Fig. 6: Comparison of Capillary Pressure in Volcanic Sand and Fine Sand for Homogeneous and Heterogeneous Porous Media 
Table 8: Numerical values of recovery rate in volcanic sand and fine sand

\begin{tabular}{|c|c|c|c|c|}
\hline \multirow{3}{*}{ Time (sec) } & \multicolumn{2}{|c|}{ Recovery rate (\%) for Volcanic Sand } & \multicolumn{2}{|c|}{ Recovery rate (\%) for Fine Sand } \\
\hline & Homogeneous & Heterogeneous & Homogeneous & Heterogeneous \\
\hline & Porous Media & Porous Media & Porous Media & Porous Media \\
\hline $1.089 \times 10^{8}$ & 6.89 & 2.47 & 5.37 & 2.06 \\
\hline $2.179 \times 10^{8}$ & 13.31 & 4.89 & 10.46 & 4.08 \\
\hline $3.268 \times 10^{8}$ & 19.28 & 7.24 & 15.27 & 6.05 \\
\hline $4.358 \times 10^{8}$ & 24.85 & 9.54 & 19.82 & 7.99 \\
\hline $5.447 \times 10^{8}$ & 30.03 & 11.78 & 24.13 & 9.89 \\
\hline $6.537 \times 10^{8}$ & 34.85 & 13.97 & 28.20 & 11.74 \\
\hline $7.626 \times 10^{8}$ & 39.34 & 16.09 & 32.06 & 13.56 \\
\hline $8.716 \times 10^{8}$ & 43.53 & 18.17 & 35.71 & 15.34 \\
\hline $9.806 \times 10^{8}$ & 47.42 & 20.20 & 39.17 & 17.09 \\
\hline $1.089 \times 10^{9}$ & 51.03 & 22.17 & 42.42 & 18.79 \\
\hline
\end{tabular}
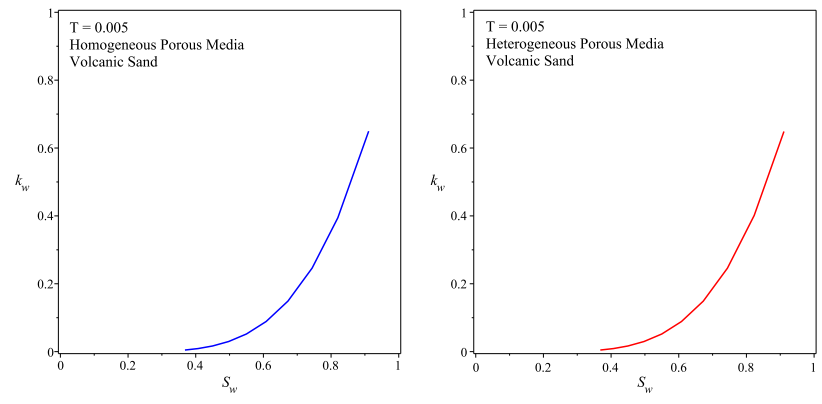

(a)

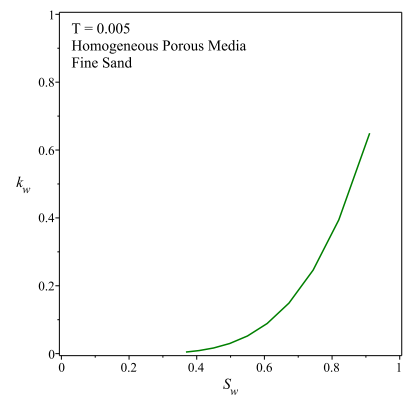

(b)

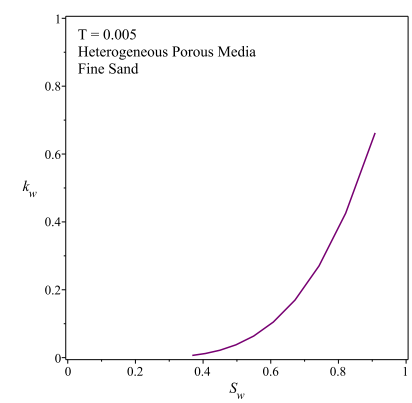

(d)

(c)

Fig. 7: Comparison between relative permeability vs. saturation in Volcanic Sand (Fig. (a) and (b)) and Fine Sand (Fig. (c) and (d))

\section{Conclusion}

Here we studied the effect of heterogeneity on saturation rate as well as on recovery rate in counter - current imbibition phenomena with the consideration of volcanic and fine sand. The simulation results for the saturation rate shown in Table 2, 3, 4 and 5 and the recovery rate of the reservoir is shown in Table 8 with the choices of suitable parametric values which shows that the saturation rate be more in volcanic sand implies the recovery rate of the oil

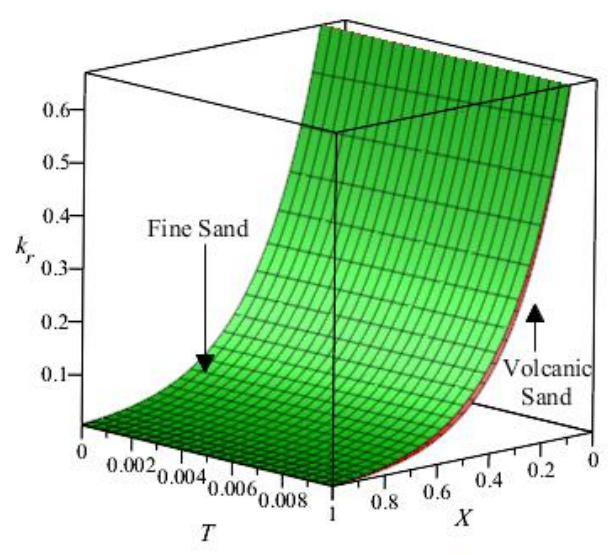

Homogeneous Porous Media

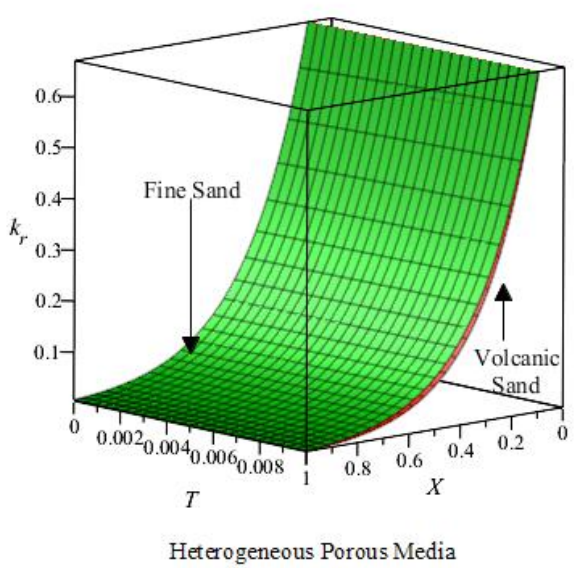

Fig. 8: Comparison of Relative Permeability in Volcanic Sand and Fine Sand for Homogeneous and Heterogeneous Porous Media

reservoir be maximum in presence of volcanic sand in homogeneous porous media and it is around $51 \%$ as com- 


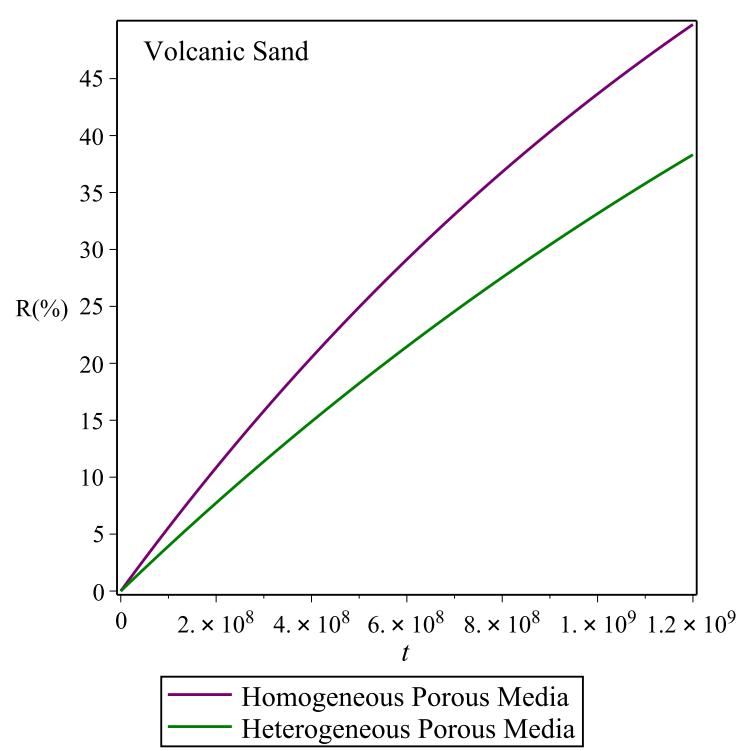

Fig. 9: Recovery rate vs.time in Volcanic Sand

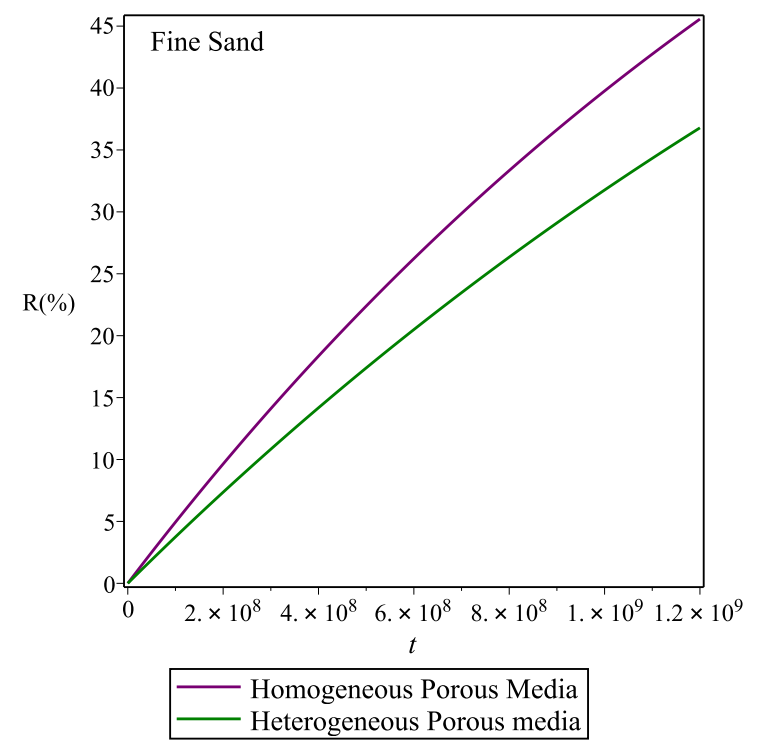

Fig. 10: Recovery rate vs. time in Fine Sand

pared to fine sand which is physically consistent with the real world phenomena.
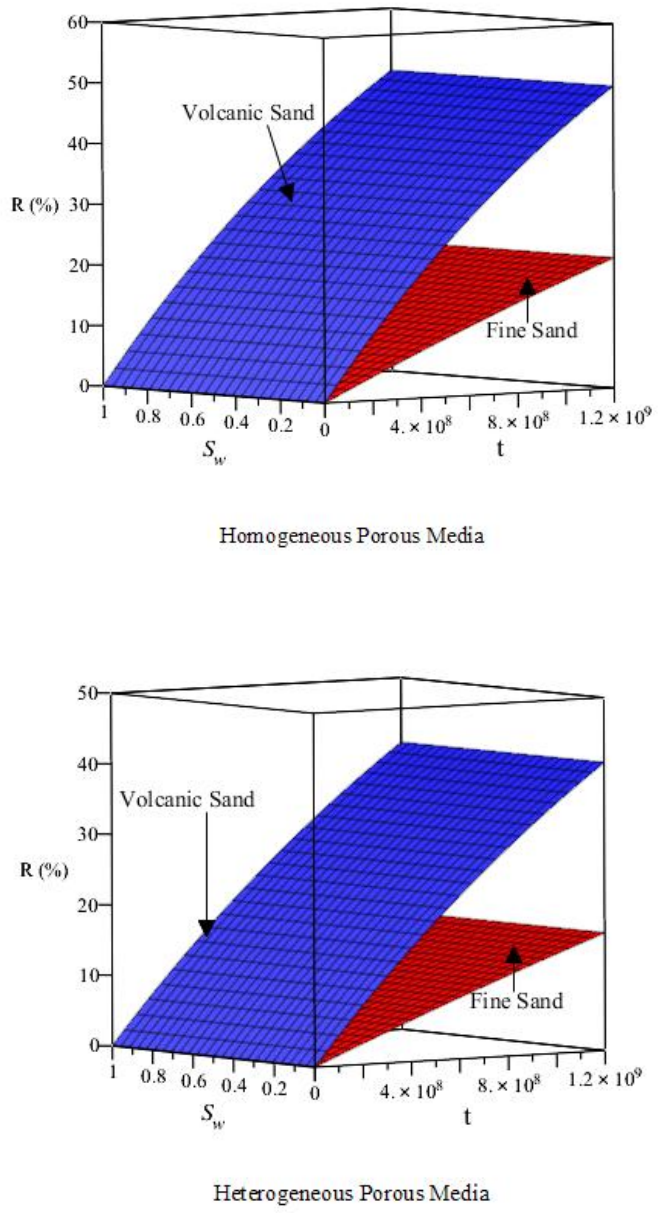

Fig. 11: Comparison of Recovery rate in Volcanic Sand and Fine Sand for Homogeneous and Heterogeneous Porous Media

\section{Nomenclature}

\begin{tabular}{cc}
\hline Symbols & Parameters \\
\hline$R$ & Recovery \\
$R_{\infty}$ & Ultimate Recovery \\
$S_{i}$ & Saturation of each phase $i$ \\
$v_{i}$ & Darcy velocity \\
$\phi$ & Porosity of the medium \\
$p_{i}$ & Pressure each phase $i$ \\
$k_{i}$ & Relative permeability of each phase $i$ \\
$\mu_{i}$ & Viscosity of each phase $i$ \\
$p_{d}$ & entry pressure \\
$S_{e}$ & effective saturation \\
\hline
\end{tabular}




\begin{tabular}{cc}
\hline Symbols & Parameters \\
\hline$\lambda$ & grain size distribution \\
$S_{w r}$ & wetting phase residual saturation \\
$K$ & Permeability \\
$p_{c}$ & Capillary Pressure \\
$\rho_{i}$ & Specific mass \\
$a, b$ and $K_{c}$ & Constants \\
$A_{n}$ & Adomian Polynomial \\
$y$ & Constant giving the rate of convergence \\
$\mu_{o}$ & Viscosity of oil \\
$\mu_{w}$ & Viscosity of water \\
$S_{w}$ & Saturation of wetting phase (water) \\
$S_{o}$ & Saturation of non - wetting phase (oil) \\
$X$ & Dimensionless Distance \\
$T$ & Dimensionless Time \\
\hline
\end{tabular}

\section{References}

[1] Adomian, G. (1994): Solving Frontier Problems of Physics: The Decomposition Method, Kluwer, Boston.

[2] Aronofsky, J. S., Masse, L., \& Natanson, S. G. (1958): A model for the mechanism of oil recovery from the porous matrix due to water invasion in fractured reservoirs, Transactions of the ASAE, 213(17), 14.

[3] Brooks, R. H., \& Corey, A. T. (1964): Hydraulic properties of porous media and their relation to drainage design, Transactions of the ASAE, 7(1), 26-0028.

[4] Hughes, R. G., \& Blunt, M. J. (2000): Pore scale modeling of rate effects in imbibition, Transport in Porous Media, 40(3), 295-322.

[5] Karpyn, Z. T., Li, G., Grader, A. S., \& Halleck, P. M. (2006): Experimental conditions favoring the formation of fluid banks during counter-current flow in porous media, Transport in porous media, 62(1), 109-124.

[6] Kashchiev, D., \& Firoozabadi, A. (2003): Analytical solutions for $1 \mathrm{D}$ counter-current imbibition in water-wet media, SPE journal, 8(04), 401-408.

[7] Meher, R., Mehta, M. N., \& Meher, S. K. (2011): A new approach to Bäcklund transformations for longitudinal dispersion of miscible fluid flow through porous media in oil reservoir during secondary recovery process, Theoretical and Applied Mechanics, 38, 1-16.

[8] Meng, Q., Liu, H., \& Wang, J. (2017): A critical review on fundamental mechanisms of spontaneous imbibition and the impact of boundary condition, fluid viscosity and wettability, Advances in Geo-energy Research, 1, 1-17.
[9] Oroveanu, T. (1963): Scurgerea fluidelor prin medii poroase neomogene, Editura Academiei Republicii Populare Romıne, 92, 328.

[10] Pooladi-Darvish, M., \& Firoozabadi, A. (2000): Co-current and counter-current imbibition in a water-wet matrix block, Spe Journal, 5(01), 3-11.

[11] Pooladi-Darvish, M., \& Firoozabadi, A. (1998): Experiments and modelling of water injection in water-wet fractured porous media, In Annual Technical Meeting. Petroleum Society of Canada, 39(3), 3-11.

[12] Patel, H. S. \& Meher, R. (2016): Fingering Phenomena in Fluid Flow Through Fracture Porous Media with Inclination and Gravitational Effect, Journal of Applied Fluid Mechanics, 9(6), 31353145.

[13] Patel, H. S., \& Meher, R. (2016): Approximate Analytical Study of Counter - Current Imbibition Phenomenon in a Heterogeneous Porous Media. Applied Mathematical Sciences, 10(14), 673-681.

[14] Patel, H. S., \& Meher, R. (2017): Modelling of Imbibition Phenomena in Fluid Flow through Heterogeneous Inclined Porous Media with different porous materials, Nonlinear Engineering, 6(4), 263-275.

[15] Patel, H. S., \& Meher, R. (2017): Simulation of Counter-Current Imbibition Phenomenon with Corey's Model in Double Phase Flow Through Heterogeneous Porous Medium with Capillary Pressure, International Journal of Applied and Computational Mathematics, 3(4), 3817-3830.

[16] Patel, K. K., Mehta, M. N., \& Singh, T. R. (2016): A homotopy series solution to a nonlinear partial differential equation arising from a mathematical model of the counter-current imbibition phenomenon in a heterogeneous porous medium, European Journal of Mechanics-B/Fluids, 60, 119-126.

[17] Saboorian-Jooybari, H., Ashoori, S., \& Mowazi, G. (2012): Development of an analytical time-dependent matrix/fracture shape factor for counter-current imbibition in simulation of fractured reservoirs, Transport in porous media, 92(3), 687708.

[18] Saboorian-Jooybari, H., Ashoori, S., \& Mowazi, G. (2015): A New Transient Matrix/Fracture Shape Factor for Capillary and Gravity Imbibition in Fractured Reservoirs, Energy Sources, Part A: Recovery, Utilization, and Environmental Effects, 37(23), 2497-2506.

[19] Zeybek, M., Gurakin, G., Donmez, A., \& Onur, M. (1995): Effects of capillary heterogeneities on spontaneous imbibition, In SPE Annual Technical Conference and Exhibition. Society of Petroleum Engineers, 779-793. 